\title{
Identification of the isoamylase 3 gene in common wheat and its expression profile during the grain-filling period
}

\author{
G.Z. Kang ${ }^{1}$, G.Q. Liu ${ }^{1}$, W. Xu ${ }^{1}$, Y.J. Zhu ${ }^{1}$, C.Y. Wang ${ }^{1}$, H.Q. Ling ${ }^{2}$ and \\ T.C. Guo ${ }^{1}$
}

${ }^{1}$ National Engineering Research Centre for Wheat,

Key Laboratory of Physiology,

Ecology and Genetic Improvement of Food Crops in Henan Province,

Henan Agricultural University, Zhengzhou, China

${ }^{2}$ The State Key Laboratory of Plant Cell and Chromosome Engineering,

Institute of Genetics and Developmental Biology,

Chinese Academy of Sciences, Chaoyang District, Beijing, China

Corresponding authors: G.Z. Kang / T.C. Guo

E-mail: zkangg@163.com / tcaiguo@163.com

Genet. Mol. Res. 12 (4): 4264-4275 (2013)

Received August 6, 2012

Accepted November 10, 2012

Published February 28, 2013

DOI http://dx.doi.org/10.4238/2013.February.28.17

\begin{abstract}
In higher plants, isoamylase-type starch debranching enzyme catalyzes the $\alpha-1,6$-glucosidic linkages of glycogen and phytoglycogen. We cloned an isoamylase-type starch debranching enzyme ISA3 cDNA sequence (2883 bp), designated as TaISA3, from common wheat (Triticum aestivum), using the rapid amplification of cDNA ends method. The open reading frame of TaISA3 was found to have $2331 \mathrm{bp}$, and its deduced amino acid sequence was found to share high similarity with those of other gramineous plant ISA3 proteins. It contains a putative transit peptide (68 amino acids), N-terminus domain (107 amino acids), and a catalytic domain (173 amino acids). We extracted the expressed TaISA3 protein from Escherichia coli (BL21), and measured starch isoamylase activity. During the wheat grain-filling period, transcripts of the TaISA3 gene
\end{abstract}


reached a maximum level at the early developmental stage, then declined, and increased again near the final maturation stage of the grain. We confirm that the $I S A 3$ gene is present in common wheat; it appears to play a role in starch synthesis during early and late stages of the grain-filling period.

Key words: Isoamylase; DNA isolation; Starch; Wheat (Triticum aestivum L.)

\section{INTRODUCTION}

Plant starch consists almost totally of glucose (Glc) homopolymers amylopectin and amylose. Amylose is a lightly branched linear molecule with low polymerization, while amylopectin is a much larger molecule with extensive branches resulting from $\alpha-1,6$ linkages (Smith et al., 1997; Dian et al., 2003). Amylose is synthesized by ADP-Glc pyrophosphorylase (AGPase) and granule-bound starch synthase (GBSS), while amylopectin is synthesized by the coordinated actions of AGPase, soluble starch synthase (SS), starch branching enzyme (BE), and starch debranching enzyme (DBE) (Han et al., 2007; Jeon et al., 2010). Disproportionating enzyme (DPE) and phosphorylase (PHO) are generally considered to be involved in starch degradation, but recent studies suggest that both DPE and PHO may play some roles in starch biosynthesis, although the precise mechanisms are unclear (Ball and Morell, 2003; Ohdan et al., 2005).

There are two classes of DBE in higher plants, and they are referred to as pullulanasetype DBE (PUL) and isoamylase-type DBE (ISA), based on similarities to prokaryotic enzymes with particular substrate specificity. Isoamylase can debranch glycogen and phytoglycogen, but barely acts on pullulan, while the reverse is true for pullulanase (Ball et al., 1996; Beatty et al., 1999). Four genes coding for DBE proteins are conserved in higher plants, one for a PUL protein and three for ISA proteins, designated as ISA1, ISA2, and ISA3 (Deschamps et al., 2008). In higher plants, ISA 1 and ISA 2 proteins exist together in a heteromeric complex, function together, and are strongly implicated in amylopectin synthesis (Fujita et al., 1999; Hussain et al., 2003; Bustos et al., 2004; Delatte et al., 2005; Utsumi and Nakamura, 2006; Takashima et al., 2007; Zeeman et al., 2010). In this complex, ISA2 is likely regulatory, because variant amino acids at essential catalytic residues of the $\alpha$-amylase superfamily render it enzymatically inactive (Hussain et al., 2003). ISA3 is not strongly associated with this multimer and may function as a monomer. Mutational analysis of ISA3 function in Arabidopsis indicates a primary role in starch breakdown but also a partially overlapping function with ISA1/ISA2 in biosynthesis (Streb et al., 2008; Wattebled et al., 2008).

Amylose is essentially a linear molecule, in which glucosyl monomers are joined via $\alpha-1,4$ linkages. Amylopectin, the more abundant polymer in starch, has a much more defined structure called 'tandem-cluster structure' compared to glycogen, because it is composed of tandem-linked clusters (approximately 9-10 nm each in length) where linear $\alpha$-1,4-glucan chains are regularly branched via $\alpha$-1,6-glucosidic linkages (Jeon et al., 2010). Variation in the cluster fine structure is considered to cause variations in starch functional properties between species, tissues, and genetic backgrounds. AGPase, GBSS, SS, BE, DBE, DPE, and PHO enzymes have multiple isoforms, the number of which is plant species specific (Ohdan et al., 2005). For example, SS, which functions in the elongation of linear glucan chains by catalyzing the transfer of the glucosyl unit of AGP-Glc to the non-reducing end of a glucan chain, has four subclasses, 
namely SSI, SSII, SSIII, and SSIV, where SSII is further divided into the three isoforms $a, b$ and c (Hirose and Terao, 2004). It has been reported that SSIIc exists in rice and Arabidopsis but not in maize (Ohdan et al., 2005). AGPase catalyzes the conversion of Glc-1-P and ATP to pyrophosphate and ADP-Glc, and is thus thought to be the rate-limiting enzyme of starch synthesis (Taliercio, 2011). AGPases in higher plants are heterotetramers composed of two large subunits (AGPL) and two small subunits (AGPS). Four AGPL isoforms (AGPL 1, AGPL 2, AGPL 3, and AGPL 4) have been identified in rice, maize and cotton (Lai et al., 2004; Ohdan et al., 2005; Taliercio, 2011). In barley, AGPL1 and AGPL2 have been identified, but AGPL 3 and AGPL 4 have not been shown to exist (Radchuk et al., 2009).

ISA3 has already been identified in rice, maize, Arabidopsis, etc. (Kubo et al., 1999; Streb et al., 2008; Yan et al., 2009). However, whether ISA3 exists in common wheat (Triticum aestivum L.) has not been reported so far. In the present study, the ISA3 gene was first isolated from common wheat using the rapid amplification of cDNA ends method (RACE), indicating that it also exists in wheat. The catalytic activity of isoamylase enzyme synthesized in Escherichia coli was measured, and ISA3 expression pattern during the grain-filling period was further investigated.

\section{MATERIAL AND METHODS}

\section{Plant material and growth conditions}

The common wheat (T. aestivum L.) cultivar Yujiao 2 was planted at the Experimental Farm of the Agricultural Faculty $\left(34^{\circ} \mathrm{N}, 113^{\circ} \mathrm{E}\right.$, and $52 \mathrm{~m}$ elevation), Henan Agricultural University during the wheat growing seasons. The status of field nutrients and plant growth and development were shown in a previous study (Kang et al., 2010).

\section{cDNA cloning and sequence analysis of the TaISA3 gene}

BLAST search of wheat databases from the Functional Genomics group in the University of Bristol (http://www.cerealsdb.uk.net/CerealsDB/Documents/DOC_search_reads.php) using the cDNA sequences of OsISA3 (GenBank accession No. NM_001069968) retrieved some wheat ESTs (contig237334, contig918142, contig1429932, etc.), which had high similarities (86-96\%) to OsISA3. Primers were designed according to these sequences to amplify the full-length cDNA sequences of TaISA3 using the BD SMART ${ }^{\mathrm{TM}}$ RACE cDNA Amplification kit (Invitrogen, Carlsbad, CA, USA). To confirm the assembled full-length sequence of the TaISA3 gene obtained with RACE, another pair of primers was designed to amplify the open reading frame (ORF) sequence of the above TaISA3 gene using the first-strand cDNA synthesized from the above extracted RNA. All primers are listed in Table 1. Five positive clones identified by PCR were sequenced.

Sequence data were analyzed using BLAST (http://www.ncbi.nlm.nih.gov/blast/), CAP3 Sequence Assembly Program (http://pbil.univ-lyon1.fr/cap3.php), and ORF Finder (http://www.ncbi.nih.gov/gorf/gorf.html). The alignment of the deduced protein sequences and phylogenetic tree were computed using DNAMAN and the ClustalX version 1.81 program employing standard parameters, respectively. TargetP version 1.1 was used to analyze the transit peptides of ISA3 proteins of wheat and other crops without cutoff, including the cleavage site prediction (http://www.cbs.dtu.dk/services/TargetP/) (Emanuelsson et al., 2007). 
expression of TaISA3 during starch synthesis. All of the developing grains from each spikelet harvested in the morning at various stages of grain filling were removed and the embryos were dissected. The sampled grains were frozen in liquid nitrogen for $2 \mathrm{~min}$ and then stored at $-80^{\circ} \mathrm{C}$ for gene expression analysis.

Semi-quantitative RT-PCR method was used to measure mRNA expression of TaISA3 gene in wheat grains during the grain-filling period. Total RNA was extracted using the Trizol reagent according to manufacturer instructions (Invitrogen). The first-strand cDNA was synthesized from $3 \mu \mathrm{g}$ total RNA using M-MLV reverse transcriptase from Promega. Each RNA sample was evaluated by the relative expression levels of the wheat $\beta$-actin gene (GenBank accession No. AB181991). All primers and the PCR programs are given in Table 1. The experiments were repeated three times.

\section{RESULTS}

\section{Isolation and characterization of the TaISA3 cDNA sequence}

Following 3'- and 5'-RACE analyses, two cDNA fragments around 1600 and $1300 \mathrm{bp}$ were detected with a $1.5 \%$ agarose gel. Following plasmid construction, transformation and cDNA clone sequencing, 1608 bp for 3'-RACE and 1304 bp for 5'-RACE fragments were obtained by removing vector and adapter sequences, respectively. The sequence of TaISA3 was deposited in GenBank and was assigned accession No. JN412069.

The full-length cDNA of TaISA3 gene was obtained by aligning and assembling the 3'and 5'-RACE sequences with the CAP3 software. To confirm its authenticity, a pair of primers (its sense primer located before the start codon ATG, and its reverse primer located after the stop codon TGA) were designed. Using this pair of primers, the predicted band was amplified with the first-strand cDNA from green grain RNA as the template, and verified by sequencing (data not shown).

The length of the amplified TaISA3 cDNA sequence was $2883 \mathrm{bp}$, containing an ORF of $2331 \mathrm{bp}$ (the region from 195 to 2525 nucleotides), flanked by 5'- and 3'-untranslated regions of 194 and $358 \mathrm{bp}$, respectively. The predicted TaISA3 protein had 776 amino acids with a calculated molecular mass of $86,112 \mathrm{Da}$ (Figure 1).

\section{Phylogenetic analysis}

The phylogenetic analysis of a total of 23 ISA proteins in higher plants was performed and indicated that ISAs were grouped into three branches: I, the ISA1 branch; II, the ISA2 branch; and III, the ISA3 branch (Figure 2). Each branch was further divided into two subbranches: monocotyledonous and dicotyledonous. In the ISA3 branch, TaISA3 was grouped into the monocotyledonous ISA3 sub-branch, and it showed $96.02,82.12$ and $76.71 \%$ similarities to HvISA3, OsISA3 and ZmISA3, respectively. However, TaISA3 shared only about $66 \%$ similarity to dicotyledonous sub-branch ISA3 proteins. This implied that the evolution of ISA3 genes in monocots had occurred after the divergence of the monocots from the dicots. Additionally, TaISA3 had less than 50\% amino acid sequence similarity to other ISA1 and ISA2 isoform proteins. This suggested that TaISA3 isolated in this study belonged to a member of the plant ISA3 family. 


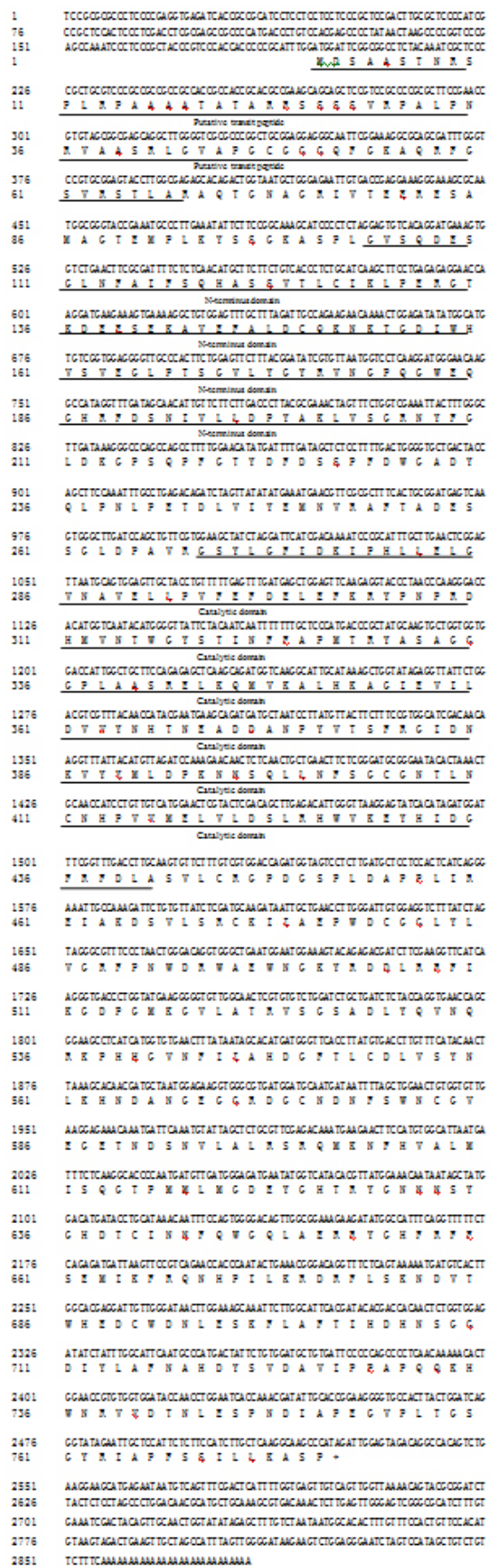

Figure 1. Nucleotide and deduced amino acid sequence of the cDNA clone TaISA3. The nucleotide sequence was presented over the deduced amino acid sequence. The translation stop codon was noted by an asterisk. Putative transit peptide, $\mathrm{N}$-terminus domain and catalytic domain were underlined and noted. 


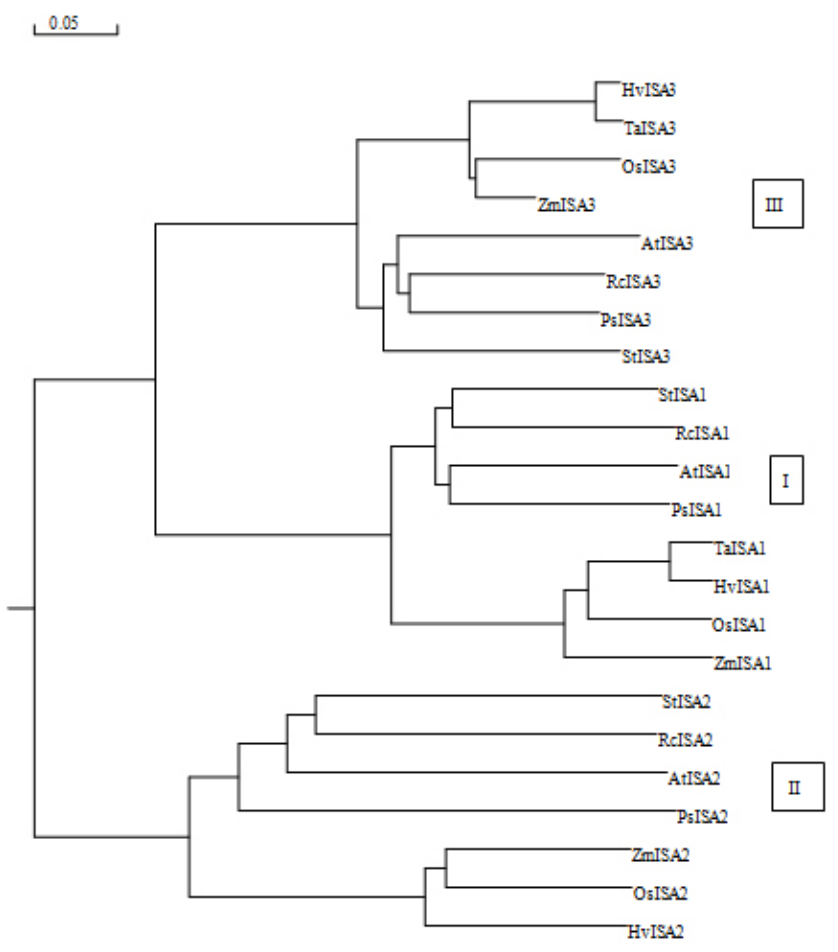

Figure 2. Phylogenetic relationship of ISA1, ISA2 and ISA3 proteins in high plants. The sequences of members were obtained from GenBank/EMBL/DDBJ database. TaISA1 and 3 (Triticum aestivum: CAC82925 and AEV92948 and 3); HvISA1, 2 and 3 (Hordeum vulgare: AAM46866, BAD08581 and BAD89532); OsISA1, 2 and 3 (Oryza sativa: ACY56095, AAT93894 and EEE69882); ZmISA1, 2 and 3 (Zea mays: AAB97167, ACG48178 and NP001105198); StISA1, 2 and 3 (Solanum tuberosum: AAM15317, AAN15318 and AAN15319); RcISA1, 2 and 3 (Ricinus communis: XP002529900, XP002533079 and XP002513977); AtISA1, 2 and 3 (Arabidopsis thaliana: NP181522, NP171830 and NP192641); PsISA1, 2 and 3 (Pisum sativum: AAZ81835, AAZ81836 and AAZ81837). TaISA2 has not been reported so far, and was not listed in this figure.

\section{Domain organization of TaISA3 proteins}

Starch in higher plants is synthesized in plastids, and important enzymes of starch synthesis, except for cytosolic AGPase in cereal crops, are located in plastids. These proteins are targeted to plastids by transit peptides at their $\mathrm{N}$ termini (Jeon et al., 2010). The TargetP software (www.cbs.dtu.dk/services/TargetP) is a good tool to predict the transit peptides and their cleavage cites for the proteins that are located in plastids, and has broadly been used so far (Bhushan et al., 2006; Emanuelsson et al., 2007; Lee et al., 2008). Prediction using the TargetP software indicated that TaISA3 contained a transit peptide of 68 amino acids (Figure 1). Its length was approximately the same as that of other higher plants, and its amino acid sequence had high similarity to that of other higher plants (data not shown).

Sequence analysis indicated that TaISA3 contained an N-terminal domain of 107 amino acids and a catalytic domain of 173 amino acids (Figure 1). In higher plants, the catalytic domains of ISA3 proteins were also divided into three types (Figure 3). TaISA3 (T. aestivum), 
HvISA3 (Hordeum vulgare), OsISA3 (Oryza sativa) and PvISA3 (Phaseolus vulgaris) belonged to type I; the lengths of their catalytic domains were about 173 amino acids, and they shared high similarities (86.13-98.84\%). AtIS3 (Arabidopsis thaliana), AlISA3 (Arabidopsis lyrata subsp) and PtISA3 (Populus trichocarpa) belonged to type II, and their catalytic domain lengths were about 139 amino acids. However, the lengths for PsISA3 (Pisum sativum), ZmISA3 (Zea mays), RcISA3 (Ricinus communis), StISA3 (Solanum tuberosum), MeISA3 (Manihot esculenta), and VvISA3 (Vitis vinifera) were only 104 amino acids and they belonged to type III (Figure 3).

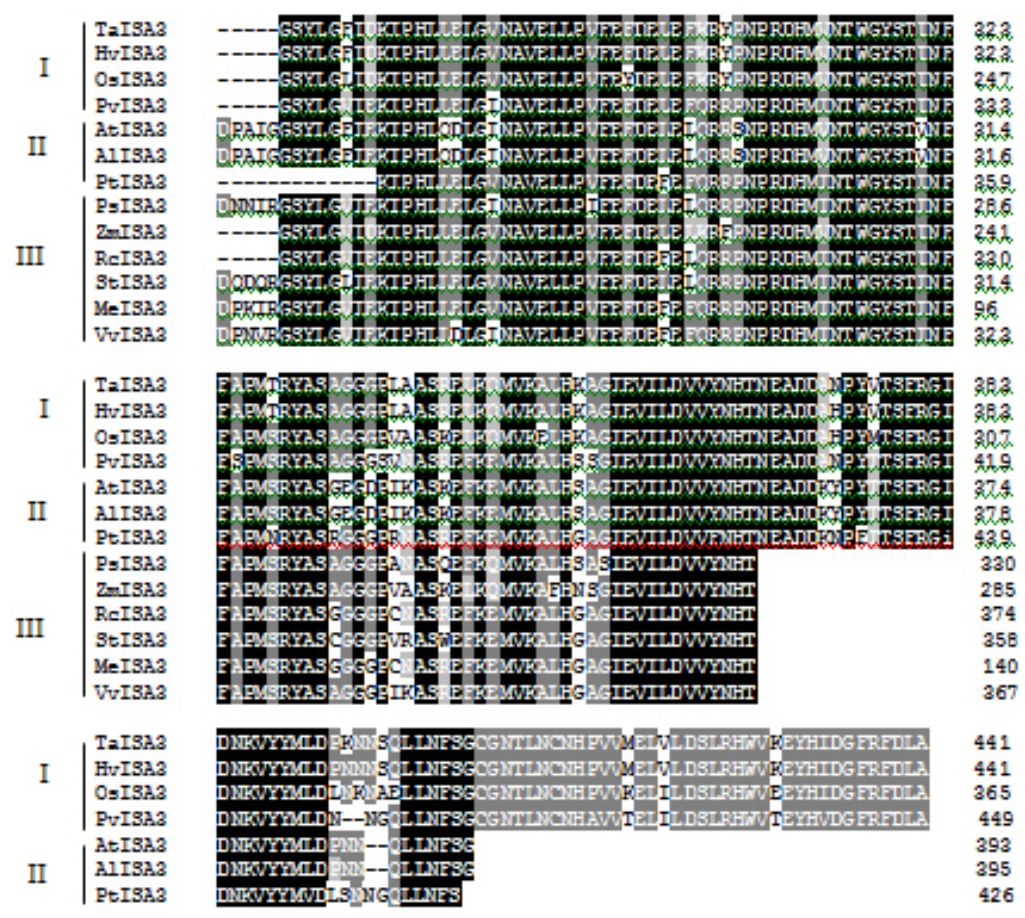

Figure 3. Alignment of the catalytic domains of ISA 3 in high plants. The sequences were aligned by the DNAMAN program. The sequences of members were obtained from GenBank/EMBL/DDBJ database. PtISA3 (Populus trichocarpa: XP_002301076), VvISA3 (Vitis vinifera: CBI19205); MeISA3 (Manihot esculenta: ADD10143); AlISA3 (Arabidopsis lyrata subsp: XP 002874494); PvISA3 (Phaseolus vulgaris: BAF52943). Other GenBank accession numbers of ISA3 were listed in Figure 2. Identical amino acid residues were shown in white, black or gray according to their similarities.

\section{Expression of the TaISA3 protein in $E$. coli and its catalytic activity}

To determine if TaISA3 gene encodes an active enzyme, its ORF sequence was cloned and inserted into the expression vector pGEXT. The expression of TaISA3 protein in E. coli (BL21) was confirmed using SDS-PAGE. The predicted size $(110 \mathrm{kDa})$ of the combined proteins, S-TAG $(24 \mathrm{kDa})$ and TaISA3 $(86 \mathrm{kDa})$, was determined (Figure 4). Measurement of the catalytic activity of TaISA3 protein showed that TaISA3 had relatively high activity on $\beta$-limit dextrin, low on amylopectin and least on phytoglycogen (Figure 5). 


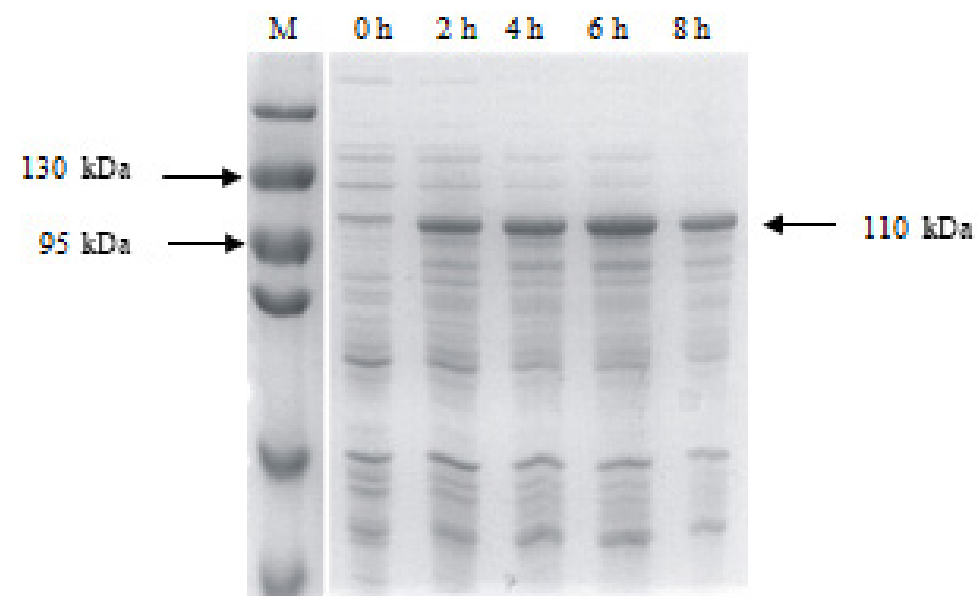

Figure 4. Expression of TaISA3 proteins in Escherichia coli (BL21). Lanes $o h$ to $8 h=$ TaISA3 proteins were induced by IPTG for $0,2,4,6$, and $8 \mathrm{~h}$. The marked bound by an arrow was the combined proteins of S-TAG (about $24 \mathrm{kDa}$ ) and TaISA3 (about $86 \mathrm{kDa}$ ). The bound proteins were separated by SDS-PAGE, and stained with Coomassie blue; lane $M=$ protein marker.

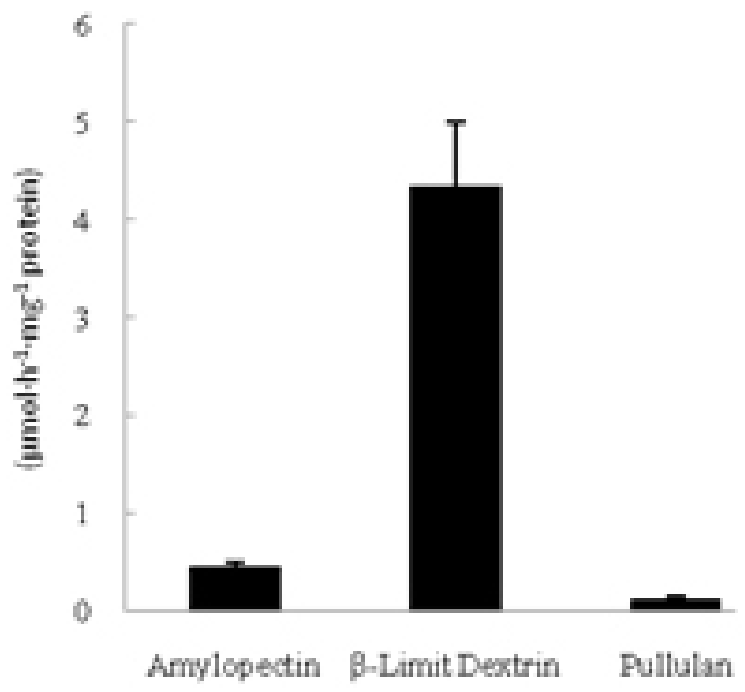

Figure 5. Activity of TaISA3 proteins extracted from Escherichia coli cell on amylopectin, $\beta$-limit dextrin and pullulan. Values are reported as means \pm standard deviation of three replications.

\section{Expression patterns of the TaISA3 gene during grain-filling period}

Starch, the primary product in wheat grains, accounts for approximately 65 to $75 \%$ of grain weight and is richly synthesized during the grain-filling period (Jeon et al., 2010). RT-PCR analysis revealed that the TaISA3 transcript was abundantly expressed at early and late stages of grain filling, and weakly at the middle developmental stage of grain filling (Figure 6). 


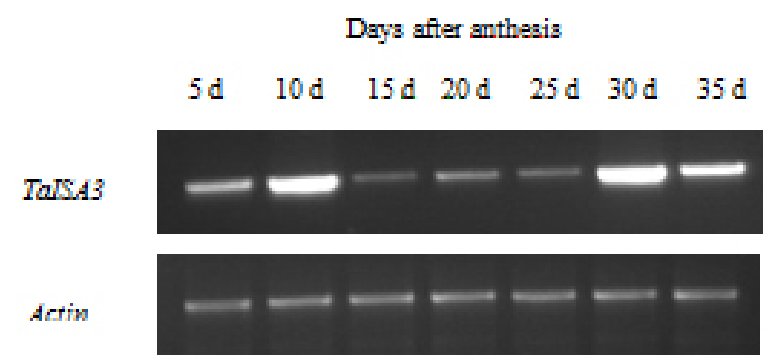

Figure 6. mRNA expression patterns of the TaISA3 gene during the grain-filling period of Yujiao 2 cultivar using semi-quantitative RT-PCR method. $\mathrm{d}=$ days.

\section{DISCUSSION}

The present study demonstrated that the ISA3 gene also exists in common wheat, and its full-length cDNA sequence was successfully isolated. First, the putative amino acids encoded by TaISA3 gene had higher similarities to HvISA3, OsISA3 and ZmISA3 than other ISA isoforms (Figure 2). Second, the TaISA3 protein contained the conserved domain characteristics of plant ISA3 proteins, including the transit peptide (Figure 1) and N-terminal and catalytic domains (Hussain et al., 2003). Its transit peptide and catalytic domain also shared higher similarities to those of HvISA3, OsISA3, etc. (Figure 3). Third, a direct test of enzyme activity illustrated that TaISA3 protein expressed in $E$. coli had relatively high activity on $\beta$-limit dextrin but much lower activity against amylopectin and phytoglycogen (Figure 5). Preference of plant ISA3 enzymes for $\beta$-limit dextrin over amylopectin has also been confirmed in potato and Arabidopsis (Hussain et al., 2003; Streb et al., 2008).

The isoforms examined so far, $\mathrm{SS}, \mathrm{BE}$, and DBE, play distinct roles in amylopectin biosynthesis. Therefore, analysis of the expression patterns of individual genes is important to understand the features of tissue-specific and developmental stage-specific starch biosynthesis (Nakamura, 2002; Ohdan et al., 2005). In this study, the TaISA3 gene expressed abundantly at early and late stages of grain filling, but weakly at the middle developmental stage of grain filling (Figure 6). This suggested that TaISA3 may play a partial role in starch synthesis during early and late stages of the grain-filling period. This was similar to the expression profile of the HvISA3 gene (Radchuk et al., 2009), but different from those of $O s I S A 3$ in rice and $\mathrm{Zm} / \mathrm{SA3}$ in maize. In rice, OsISA3 has a comparatively low transcript level at the onset of grain development, and expression remains almost constant through the late-milking stage of grain development (Ohdan et al., 2005), whereas ZmISA3 in maize is mainly expressed in leaves and early developing endosperm (Yan et al., 2009). This suggests that, in starch metabolism, the role of TaISA3 could be similar to that of HvISA3, but different from that of OsISA3 and ZmISA3. This, however, needs to be confirmed by further studies.

\section{ACKNOWLEDGMENTS}

Research supported by the National Natural Science Foundation of China (\#31171471 and \#30871472), the State Key Laboratory of Crop Biology (\#2009KF01), the State Key Laboratory of Plant Cell and Chromosome Engineering (\#2010-PCCE-KF-02), and the State Key 
Laboratory of Crop Genetics and Germplasm Enhancement (\#ZW2009003). We thank Dr. Shusong Zheng for valuable suggestions on the manuscript's modification.

\section{REFERENCES}

Ball SG and Morell MK (2003). From bacterial glycogen to starch: understanding the biogenesis of the plant starch granule. Annu. Rev. Plant Biol. 54: 207-233.

Ball SG, Guan HP, James M, Myers A, et al. (1996). From glycogen to amylopectin: a model for the biogenesis of the plant starch granule. Cell 86: 349-352.

Beatty MK, Rahman A, Cao H, Woodman W, et al. (1999). Purification and molecular genetic characterization of ZPU1, a pullulanase-type starch-debranching enzyme from maize. Plant Physiol. 119: 255-266.

Bhushan S, Kuhn C, Berglund AK, Roth C, et al. (2006). The role of the N-terminal domain of chloroplast targeting peptides in organellar protein import and miss-sorting. FEBS Lett. 580: 3966-3972.

Bustos R, Fahy B, Hylton CM, Seale R, et al. (2004). Starch granule initiation is controlled by a heteromultimeric isoamylase in potato tubers. Proc. Natl. Acad. Sci. U. S. A. 101: 2215-2220.

Delatte T, Trevisan M, Parker ML and Zeeman SC (2005). Arabidopsis mutants Atisa1 and Atisa2 have identical phenotypes and lack the same multimeric isoamylase, which influences the branch point distribution of amylopectin during starch synthesis. Plant J. 41: 815-830.

Deschamps P, Moreau H, Worden AZ, Dauvillee D, et al. (2008). Early gene duplication within chloroplastida and its correspondence with relocation of starch metabolism to chloroplasts. Genetics 178: 2373-2387.

Dian WM, Jiang HW, Chen QS, Liu FY, et al. (2003). Cloning and characterization of the granule-bound starch synthase II gene in rice: gene expression is regulated by the nitrogen level, sugar and circadian rhythm. Planta 218: 261-268.

Emanuelsson O, Brunak S, von Heijne G and Nielsen H (2007). Locating proteins in the cell using TargetP, SignalP and related tools. Nat. Protoc. 2: 953-971.

Fujita N, Kubo A, Francisco PB Jr, Nakakita M, et al. (1999). Purification, characterization, and cDNA structure of isoamylase from developing endosperm of rice. Planta 208: 283-293.

Han Y, Sun FJ, Rosales-Mendoza S and Korban SS (2007). Three orthologs in rice, Arabidopsis, and Populus encoding starch branching enzymes (SBEs) are different from other SBE gene families in plants. Gene 401: 123-130.

Hirose $\mathrm{T}$ and Terao T (2004). A comprehensive expression analysis of the starch synthase gene family in rice (Oryza sativa L.). Planta 220: 9-16.

Hussain H, Mant A, Seale R, Zeeman S, et al. (2003). Three isoforms of isoamylase contribute different catalytic properties for the debranching of potato glucans. Plant Cell 15: 133-149.

Jeon JS, Ryoo N, Hahn TR, Walia H, et al. (2010). Starch biosynthesis in cereal endosperm. Plant Physiol. Biochem. 48: 383-392.

Kang GZ, Wang YH, Liu C, Shen BQ, et al. (2010). Difference in AGPase subunits could be associated with starch accumulation in grains between two wheat cultivars. Plant Growth Regul. 61: 61-66.

Kubo A, Fujita N, Harada K, Satoh H, et al. (1999). The starch-debranching enzyme isoamylase and pullulanase are both involved in amylopectin biosynthesis in rice endosperm. Plant Physiol. 121: 399-409.

Lai J, Dey N, Kim CS, Bharti AK, et al. (2004). Characterization of the maize endosperm transcriptome and its comparison to the rice genome. Genome Res. 14: 1932-1937.

Lee DW, Kim JK, Lee S and Choi S (2008). Arabidopsis nuclear-encoded plastid transit peptides contain multiple sequence subgroups with distinctive chloroplast-targeting sequence motifs. Plant Cell 20: 1603-1622.

Nakamura Y (2002). Towards a better understanding of the metabolic system for amylopectin biosynthesis in plants: rice endosperm as a model tissue. Plant Cell Physiol. 43: 718-725.

Ohdan T, Francisco PB Jr, Sawada T, Hirose T, et al. (2005). Expression profiling of genes involved in starch synthesis in sink and source organs of rice. J. Exp. Bot. 56: 3229-3244.

Radchuk VV, Borisjuk L, Sreenivasulu N, Merx K, et al. (2009). Spatiotemporal profiling of starch biosynthesis and degradation in the developing barley grain. Plant Physiol. 150: 190-204.

Smith AM, Denyer K and Martin C (1997). The synthesis of the starch granule. Annu. Rev. Plant Physiol. Plant Mol. Biol. 48: 67-87.

Streb S, Delatte T, Umhang M, Eicke S, et al. (2008). Starch granule biosynthesis in Arabidopsis is abolished by removal of all debranching enzymes but restored by the subsequent removal of an endoamylase. Plant Cell 20: 3448-3466.

Takashima Y, Senoura T, Yoshizaki T, Hamada S, et al. (2007). Differential chain-length specificities of two isoamylasetype starch-debranching enzymes from developing seeds of kidney bean. Biosci. Biotechnol. Biochem. 71: 2308-2312.

Taliercio E (2011). Characterization of an ADP-glucose pyrophosphorylase small subunit gene expressed in developing 
cotton (Gossypium hirsutum) fibers. Mol. Biol. Rep. 38: 2967-2973.

Utsumi Y and Nakamura Y (2006). Structural and enzymatic characterization of the isoamylase1 homo-oligomer and the isoamylase1-isoamylase2 hetero-oligomer from rice endosperm. Planta 225: 75-87.

Wattebled F, Planchot V, Dong Y, Szydlowski N, et al. (2008). Further evidence for the mandatory nature of polysaccharide debranching for the aggregation of semicrystalline starch and for overlapping functions of debranching enzymes in Arabidopsis leaves. Plant Physiol. 148: 1309-1323.

Yan HB, Pan XX, Jiang HW and Wu GJ (2009). Comparison of the starch synthesis genes between maize and rice: copies, chromosome location and expression divergence. Theor. Appl. Genet. 119: 815-825.

Zeeman SC, Kossmann J and Smith AM (2010). Starch: its metabolism, evolution, and biotechnological modification in plants. Annu. Rev. Plant Biol. 61: 209-234. 